\title{
Feasible Multiple Satellite Mission Scenarios Flying in a Constellation for Refinement of the Gravity Field Recovery
}

\author{
Basem Elsaka ${ }^{1,2}$ \\ ${ }^{1}$ King Abdulaziz City for Science and Technology (KACST), Riyadh, Saudi Arabia \\ ${ }^{2}$ National Research Institute of Astronomy and Geophysics (NRIAG), Helwan, Cairo, Egypt \\ Email: basem_elsaka2002@yahoo.com
}

Received 3 January 2014; revised 5 February 2014; accepted 4 March 2014

Copyright (C) 2014 by author and Scientific Research Publishing Inc.

This work is licensed under the Creative Commons Attribution International License (CC BY). http://creativecommons.org/licenses/by/4.0/

(c) (7) Open Access

\begin{abstract}
Improving the gravity field recovery in terms of error levels and more isotropic noise distribution by adding cross-track and radial information to the satellite observables has been investigated through a number of studies by a variety of satellite constellations, i.e. satellite pairs that orbit the Earth in alternative configurations than the current GRACE (Gravity Recovery And Climate Experiment) gravity mission. This contribution gives for the first time a comparative study considering the recovery of the global gravity field from three constellations flying in satellite pairs in different directions (i.e. along-track, cross-track and radial). The three constellations include: 1) Foursatellite Bender configuration (flying in two pairs) of type along-track observations, 2) Three-satellite GRAPEN (combined GRACE with Pendulum formations) configuration of type cross-alongtrack observations, 3) Four-satellite Cartwheel configuration (flying in two pairs) of type radialalong-track observations. Additionally, a GRACE mission scenario is added as a reference "comparative" mission. The orbits of all satellites are considered to fly with drag-free system, however, realistic white noise has been added to the simulated observations to mimic the error associated with the drag-free measurement. The results are analyzed in the spectral wavelength spectrum of the gravity field up to a spherical harmonics degree of $n=100$ and are plotted spatially on earth maps. The results show that the Three-satellite GRAPEN constellation provides, besides its low economically launches, an improved gravity field solution with respect to the Four-satellite Bender and the Four-satellite Cartwheel constellations.
\end{abstract}

\section{Keywords}

Satellite Geodesy; Multi-Satellite Constellations (Bender; Cartwheel-4S; GRAPEN); Gravity Field Recovery 


\section{Introduction}

Various studies have been published in the last years concerning the concepts for future gravity field satellite missions that will be flying beyond the era of the successful GRACE mission, e.g. by [1]-[8]. In addition, three recent studies have studied the capability of single and multiple inline formation mission scenarios with identical and difference. Two of these studies were funded by ESA (European Space Agency) [9] [10], and one was funded by the BMBF (German Federal Ministry of Education and Research) and the DFG (German Research Foundation) [11]. All these studies have had a common conclusion that the additional information expected from future alternative configuration, different from the GRACE mission, will definitely provide a significant improvement in accuracy and sensitivity of the earth's gravity field. A most recent study by [8] has concluded that the Bender configuration [2], consisting of one polar satellite pair coupled with another inclined pair of type along-track observations, and the pendulum satellite formation of type cross-track observations are possible options to the next generation gravity missions. However, the former "Bender" type is preferred if the budget allows for the launch of two satellite pairs, whereas if the budget allows only for the launch of one pair, then the latter pendulum is proposed. However, the accuracy of the one pendulum satellite pair is relatively worse with respect to (w.r.t.) the accuracy gained from the Bender configuration.

In this contribution, we compare for the first time between three multi-satellite configurations: the Four-satellite Bender constellation, which was firstly suggested by [2], the Three-Satellite GRAPEN (combined GRACE with PENDULUM formation flights) constellation, which was firstly suggested by [12] (cf. GRACE-Pendulum) and the Four-satellite Cartwheel (Cartwheel-4S in the following, oriented east-west cartwheel coupled with oriented north-south cartwheel). The idea of the Cartwheel formation was firstly proposed by [13], while the oriented north-south cartwheel has been suggested by [14]. We have to mention that these satellite constellations have been investigated for the gravity field recovery (see e.g. [15] for the Bender, [1] and [4] for the two and four Cartwheel, respectively, and [6] and [12] for the GRAPEN). They have found that an improved solution in the gravity field analysis is expected from these mission configurations.

In the following, we briefly introduce the considered three configuration options along with their orbital characteristics (Section 2). The methodology of the full-scale simulation is described in Section 3. Gravity field results are discussed in Section 4. Finally, a general conclusion is outlined in Section 5.

\section{Configuration Options}

At first, the orbital altitude for all satellite orbits is set up at $400 \mathrm{~km}$ (i.e. the orbits have an identical semi-major axis) in order to fairly compare between the gravity field solutions. A semi-polar inclination of $89.5^{\circ}$ has been selected for all orbits as same as the real GRACE mission, whereas an inclination of $63^{\circ}$ has been selected for the second Bender "inclined” satellite pair. The eccentricity has been selected to be 0.003688 for all orbits. The reason for selecting this eccentricity is due to that the inter-satellite distance in case of the Cartwheel condition is governed by the relation given by [3] as $\rho_{\min }=2$ ae or $\rho_{\max }=4 \mathrm{ae}$, where $\rho_{\min }$ and $\rho_{\max }$ are the minimum and maximum inter-satellite distances of the Cartwheel formation, respectively. An average satellite distance of 100 $\mathrm{km}$ has been selected for all baselines of the satellite configurations, whereas the inter-satellite distances for both satellite pairs of the Cartwheel configuration, due to the above selected eccentricity, range between $50 \mathrm{~km}$ and $100 \mathrm{~km}$.

Compared to the simple GRACE leader follower formation (Figure 1(a)), the Bender configuration (Figure $\mathbf{1 ( b )})$ is composed of double "polar (here $89.5^{\circ}$ ) and inclined $\left(63^{\circ}\right)$ ” collinear satellite pairs [2]. The Bender configuration is characterized by that a sufficient spatial sampling will be achieved at low latitudes due to the dense satellite tracks of the second "inclined” satellites pair $\left(63^{\circ}\right)$. Additionally, an East-West information will be added to the observables because of the second inclined satellite pair. This would increase the isotropy of gravity field solution, and hence, should reduce the longitudinal striping pattern as well as aliasing errors that are appeared in the GRACE gravity solutions (see e.g. [5] [8] [16]).

When we add another satellite with different angles of the right ascension of ascending nodes (RAAN) to the GRACE twin-satellites, the so-called GRAPEN is formed (we refer here to [6] for more details). The maximum cross-track sensitivity of the GRAPEN configuration takes place at the equator, where the distance is greatest (between GRAPEN A and GRAPEN C satellites, see Figure 1(d)). At the poles, a minimum distance between GRAPEN A and GRAPEN C takes place yielding minimum along-track sensitivity, but the sensitivity would be already supported by the maximum along-track distance between GRAPEN A and GRAPEN B. It has been 


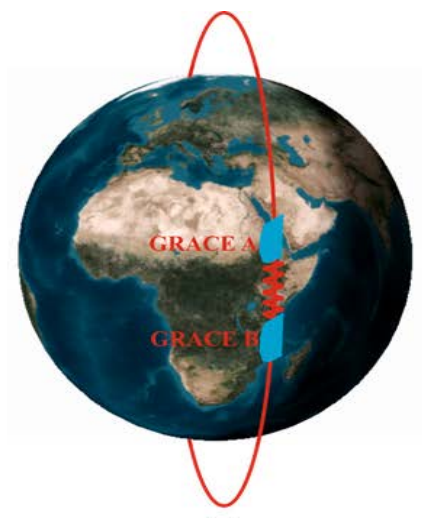

(a)

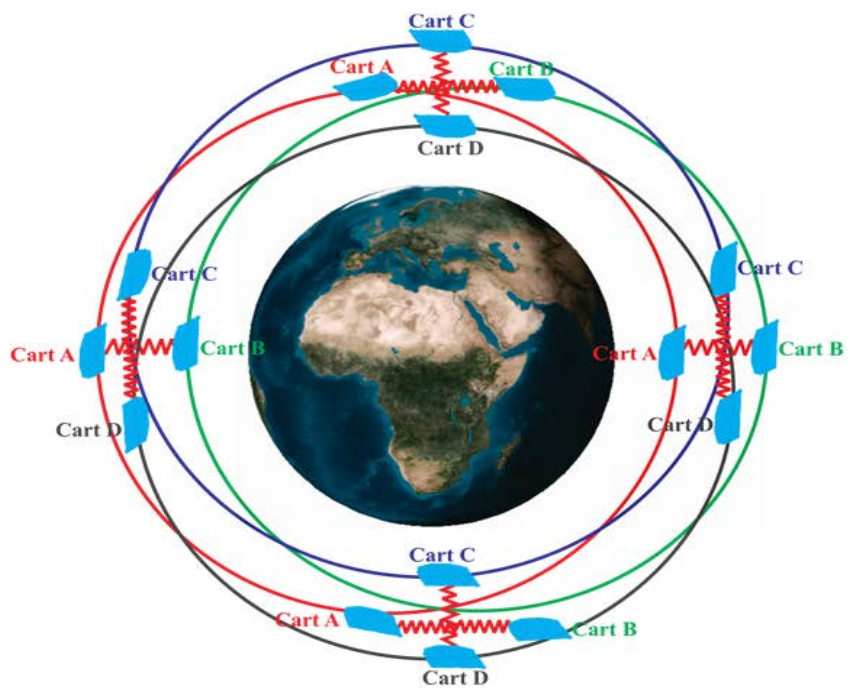

(c)

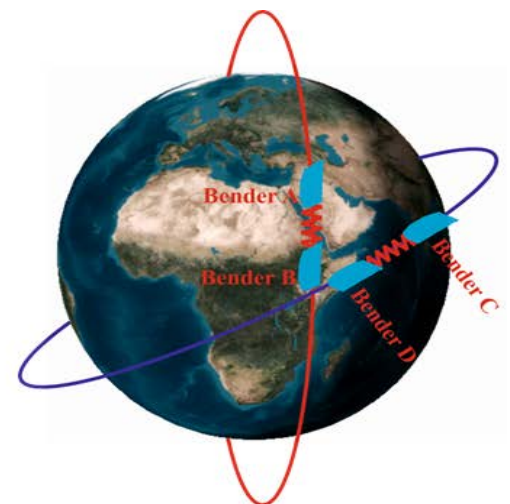

(b)

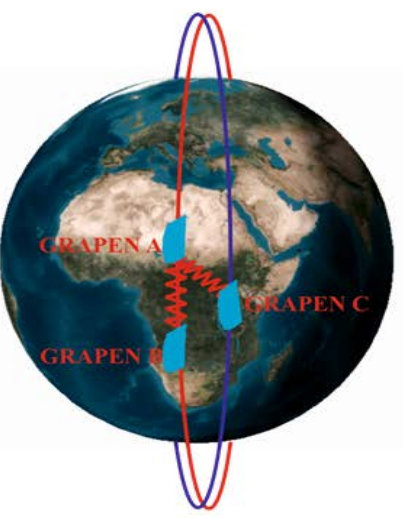

(d)

Figure 1. Multi-satellite constellations; (b) Bender, (c) Cartwheel-4S and (d) GRAPEN, compared with (a) the GRACE simple formation.

suggested by [6] and [8] that the cross-track angle between the pendulum satellites should be selected not large to avoid the high relative velocity between both cross-track satellites. Therefore, a RAAN angle of about $0.405^{\circ}$ has been selected for the GRAPEN C satellite to maintain the velocity within $\pm 10 \mathrm{~m} / \mathrm{s}$ relative to the GRAPEN A satellite. Additionally, a different mean anomaly $\left(\triangle \mathrm{M}=0.81^{\circ}\right)$ is set up between GRAPEN A and GRAPEN B as well as GRAPEN A and GRAPEN C satellites to perform the along-track distance between the satellites.

Regarding to the Cartwheel formation (Figure 1(c)), the both satellite pairs perform a 2:1 relative elliptical motion. This means that the length of the semi-major axis is twice the length of the semi-minor one about their joint center of mass. The inter-satellite range of the along-track direction is twice of the radial one. The Cartwheel-4S (here referred to as Cart A, Cart B, Cart C and Cart D) configuration is performed by setting all orbit parameters identical except for the argument of perigee $(\omega)$ and the mean anomaly $(\mathrm{M})$ by $180^{\circ}$ in a way that $\omega_{\mathrm{A}}=0^{\circ}, \mathrm{M}_{\mathrm{A}}=90^{\circ}$ and $\omega_{\mathrm{B}}=-180^{\circ}, \mathrm{M}_{\mathrm{B}}=-90^{\circ}$, whereas $\omega_{\mathrm{C}}=270^{\circ}, \mathrm{M}_{\mathrm{C}}=90^{\circ}$ and $\omega_{\mathrm{D}}=90^{\circ}, \mathrm{M}_{\mathrm{D}}=-90^{\circ}$, where subscript symbols A, B, C and D stand for Cart A, Cart B, Cart C and Cart D satellites (Figure 1(c)). The advantage of this configuration is that both along-track and radial components are taking place at both equators and poles. The along-tack component takes place at equators via satellites Cart $\mathrm{C}$ and $\mathrm{D}$ and at poles via Cart $\mathrm{A}$ and Cart B, whereas the radial component takes place at equator via satellites Cart A and B and at poles via Cart $\mathrm{C}$ and Cart D.

The selected inter-satellite distance between Cart A and Cart B and between Cart C and Cart D is set as $50 \mathrm{~km}$ for the minimum distance (i.e. in the radial direction) and as $100 \mathrm{~km}$ for the maximum distance (i.e. in the along-track direction). However, one should mention here that the relative velocities between the Cartwheel sa- 
tellites are ranging between $\pm 50 \mathrm{~m} / \mathrm{s}$, which make the mission technically challenging.

\section{Methodology}

Full-scale numerical simulations of the investigated satellite constellations were performed via two main steps; integration step and gravity analysis step. In the integration step, simulated satellite orbits of each satellite formation were integrated over a time span of one month. Measurement noise was added to the error-free observations by using a random Gaussian noise generator with standard deviation equal to $1 \mu \mathrm{m} / \mathrm{s}$ in range-rate for GRACE and $50 \mathrm{~nm} / \mathrm{s}$ for the other three configurations. We have to mention that the investigated configurations have been considered within this paper as drag-free missions, which don't carry accelerometer data; however, simulated accelerometer noise has been created of level $1 \times 10^{-10} \mathrm{~m} / \mathrm{s}^{2}$ assuming that this noise level is the error associated with the drag-free measurement.

In the gravity analysis step, we used the satellite observations (obtained from the first step) of the three configurations besides the GRACE scenario to estimate the gravity field parameters, We consider in this study the so-called "static scenario", where satellite noisy measurements were set up and used in the observation equations to produce the gravity field parameters in terms of spherical harmonics up to degree/order 100/100. The numerical simulation procedure applied in this paper is identical to that described by [6], where the majority of the details are illustrated using the GROOPS software [17] developed in the Institute of Geodesy and Geoinformation of the Bonn University.

\section{Results and Discussion}

In the following, the gravity field solutions determined from the investigated satellite configurations are discussed. In the spectral domain, the results are shown in terms of the difference degree variances of geoid heights that have been often used to quantify the powers of signal and error in the gravity field estimates at various spatial wavelengths. In the spatial domain, the results are plotted as geoid error maps.

Figure 2 (solid curves) gives the difference degree variance of geoid heights between the estimated spherical coefficients and the reference ones (from the reference model ITGGRACE03s [18]) as well as in terms of cumulative geoid errors (see Figure 2, dashed curves). Additionally, Figure 3 represents the results in terms of geoid errors on Earth's maps. As seen from Figure 2 that the investigated three constellations perform superior to the GRACE solution which confirm the findings of the previous studies (see Section 1). The GRAPEN configuration apparently provides the overall best solution with root mean square geoid error (RMS) of $0.597 \mathrm{~mm}$ as given in Table 1. This is approximately more than a full order of magnitude, in the spectral domain (Figure 2), better compared to the GRACE result of RMS $14.03 \mathrm{~mm}$. The Bender and Cartwheel-4S solutions have RMS of 0.714 and $0.699 \mathrm{~mm}$, respectively, outperforming the GRACE solution by a factor of two.

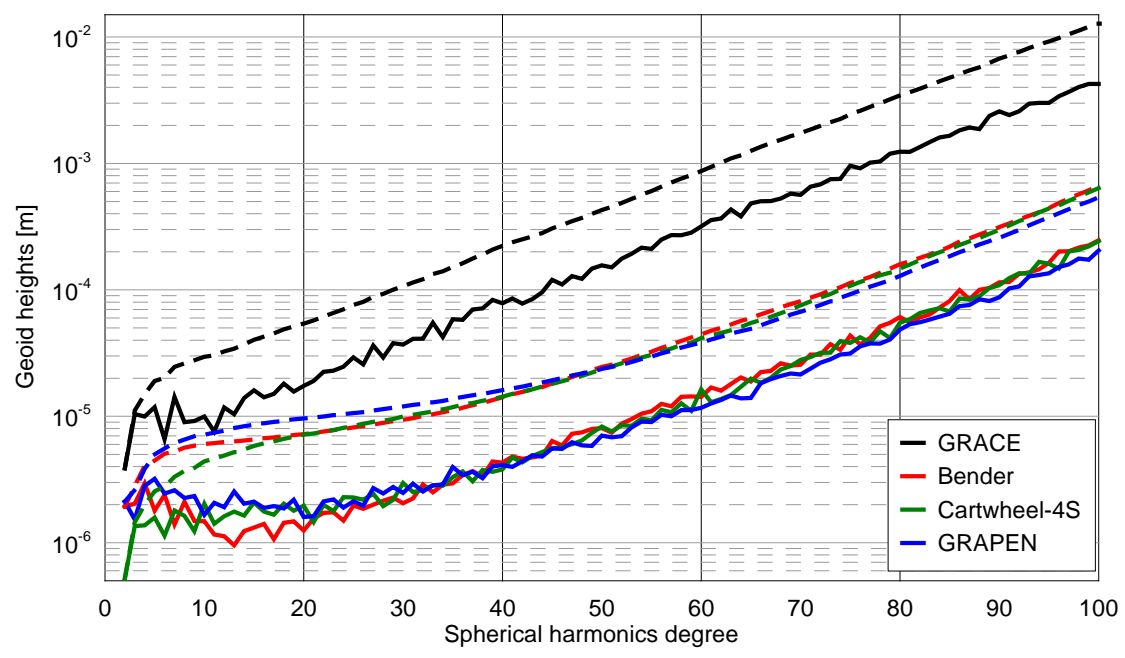

Figure 2. Gravity field solutions in terms of difference degree variance (solid curves) and cumulative geoid errors (dashed curves). 


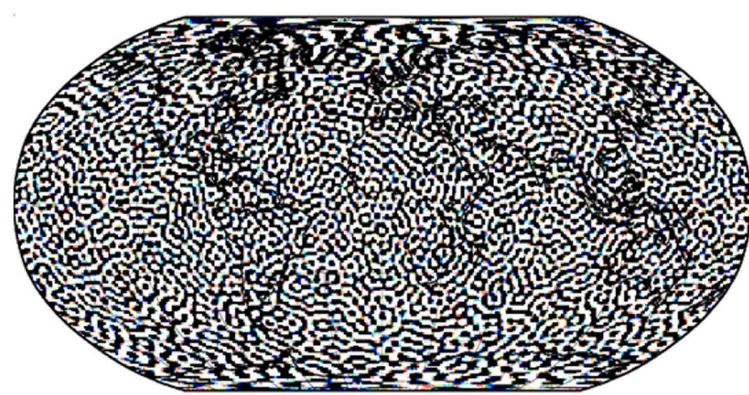

(a)

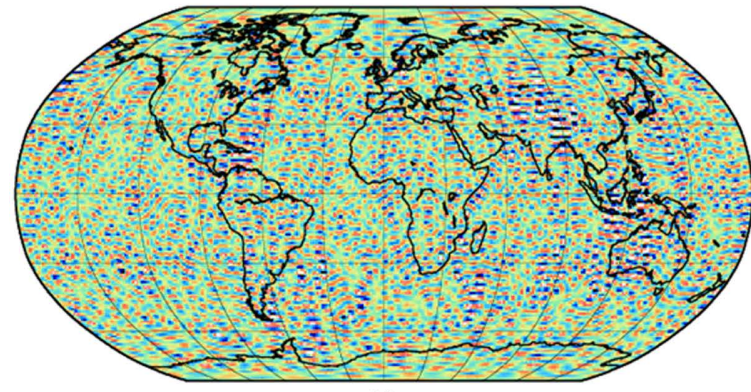

(c)

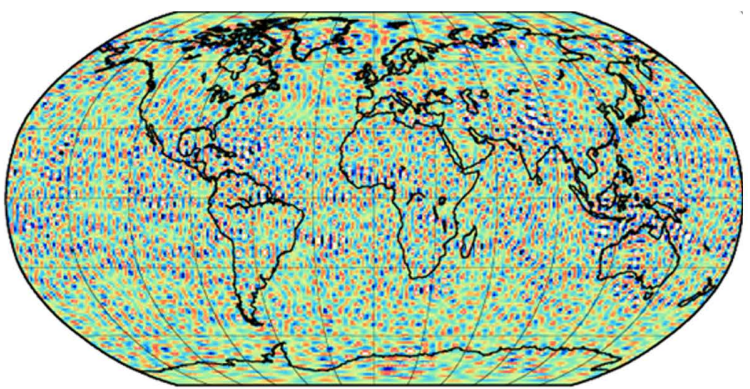

(b)

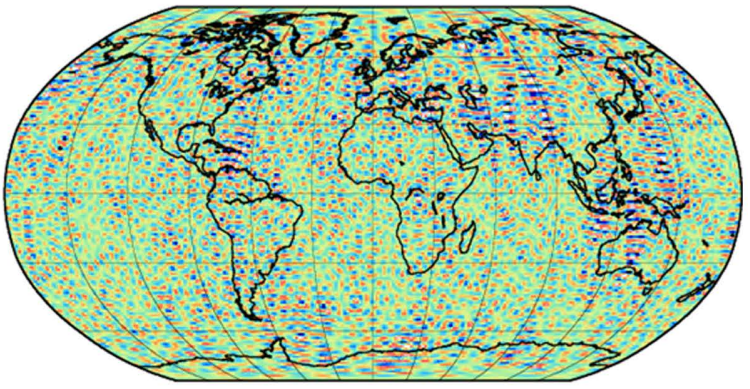

(d)

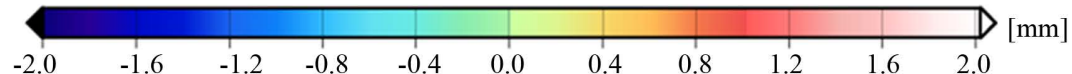

Figure 3. Gravity field solutions in terms of geoid errors as determined from (a) GRACE, (b) Bender, (c) Cartwheel-4S and (d) GRAPEN constellations.

Table 1. Geoid statistical values (RMS, minimum and maximum) of the different gravity solutions as determined by the investigated constellations up to SH degree $n=100$.

\begin{tabular}{lcccc} 
& \multicolumn{1}{l}{$\begin{array}{l}\text { Geoid } \\
\text { values [mm] }\end{array}$} & \multicolumn{4}{c}{ Gravity recovery } \\
\cline { 2 - 5 } Constellation & RMS & Avg. & Min. & Max. \\
& 14.03 & 11.08 & -57.4 & 63.4 \\
GRACE & 0.714 & 0.559 & -3.47 & 3.84 \\
Bender & 0.699 & 0.551 & -3.53 & 3.33 \\
Cartwheel-4S & 0.597 & 0.465 & -3.15 & 2.87 \\
GRAPEN & & & & \\
\hline
\end{tabular}

The gravity field solutions of the three configurations, except the GRACE, provide isotropic geoid errors as one can see in Figure 3. Taking into account that the Cartwheel-4S configuration consists of four satellites with large relative velocity (Section 2) which makes the mission technically challenging, one can consider the Bender and GRAPEN configurations as the feasible missions providing the best overall solutions. However, the Bender configurations may definitely need two launchers to carry the two satellite pairs in two different inclinations, whereas, the GRAPEN configuration may require only one launcher, since its three satellites have no change in orbital inclination, and hence, would be of a low cost w.r.t. the Bender configuration. Therefore, it would be preferred to further the investigation towards the recovery of gravity field from the GRAPEN as a feasible future mission.

\section{Conclusions}

In this study, a comparison between the recovered solutions determined by three different satellite constellations; the Bender, Cartwheel-4S and GRAPEN has been done. We confirm the findings of previous studies that 
changing the spatial sampling (with keeping the temporal sampling as identical as real GRACE mission) via these configurations could enhance the gravity field recovery and could reduce the striping pattern associated with the GRACE solutions.

Regarding to the Cartwheel-4S, the mission seems technically challenging because of its high relative velocity between Cart A and Cart B and between Cart C and Cart D which exceeds the velocities recommended by the previous studies, and hence, this mission seems difficult to be applied for the gravity field. However, the Cartwheel-4S solution provides a better gravity field solution w.r.t. the Bender configuration.

We can conclude that the Three-satellite GRAPEN configuration provides the overall best performance w.r.t. the Four-satellite Bender and the Four-satellite Cartwheel configurations. Moreover, the GRAPEN would need only one launcher to carry the Three-satellites with the same inclination which seems economic from the budget point of view w.r.t the Bender and the Cartwheel-4S, which definitely require two launchers to carry the two satellite pairs in two different inclinations.

Therefore, it would be preferred to do further investigations towards the recovery of gravity field from the GRAPEN as a feasible future mission considering in details the technical and systematic issues.

\section{Acknowledgements}

The author would like to thank Prof. Dr. Jürgen Kusche at the Institute of Geodesy and Geoinformation (IGG) of Bonn University for providing the access to GROOPS (Gravity Recovery and Object Oriented Programming System) software to perform the numerical simulations. The financial support of the King Abdulaziz City for Science and Technology (KASCT) in Riyadh, Saudi Arabia, is gratefully acknowledged.

\section{References}

[1] Sharifi, M., Sneeuw, N. and Keller, W. (2007) Gravity Recovery Capability of Four Generic Satellite Formations. In A. Kilicoglu and R. Forsberg, Eds., Gravity Field of the Earth. General Command of Mapping, S18, 211-216.

[2] Bender, P.L., Wiese, D. and Nerem, R.S. (2008) A Possible Dual-GRACE Mission with 90 Degree and 63 Degree Inclination Orbits. Proceedings of the 3rd International Symposium on Formation Flying, Missions and Technologies, ESA/ESTEC, Noordwijk, 23-25 April 2008, 1-6.

[3] Sneeuw, N., Sharifi, M. and Keller, M. (2008) Gravity Recovery from Formation Flight Missions. In: P. L. Xu, J. N. Liu and A. Dermanis, Eds., VI Hotine-Marussi Symposium on Theoretical and Computational Geodesy, 132, 29-34. http://dx.doi.org/10.1007/978-3-540-74584-6_5

[4] Wiese, D., Folkner, W. and Nerem, R. (2009) Alternative Mission Architectures for a Gravity Recovery Satellite Mission. Journal of Geodesy, 83, 569-581.

[5] Wiese, D., Nerem, R. and Han, S.-C. (2011) Expected Improvements in Determining Continental Hydrology, Ice Mass Variations, Ocean Bottom Pressure Signals, and Earthquakes Using Two Pairs of Dedicated Satellites for Temporal Gravity Recovery. Journal of Geophysical Research, 116, 405. http://dx.doi.org/10.1029/2011JB008375

[6] Elsaka, B., Kusche, J. and Ilk, K.-H. (2012) Recovery of the Earth’s Gravity Field from Formation-Flying Satellites: Temporal Aliasing Issues. Advances in Space Research, 50, 1534-1552. http://dx.doi.org/10.1016/j.asr.2012.07.016

[7] Iran Pour, S., Reubelt, T. and Sneeuw, N. (2013) Quality Assessment of Sub-Nyquist Recovery from Future Gravity Satellite Missions. Journal of Advances in Space Research, 52, 916-929. http://dx.doi.org/10.1016/j.asr.2013.05.026

[8] Elsaka, B., Raimondo, J.-C., Brieden, Ph., Reubelt, T., Kusche, J., Flechtner, F., Iran Pour, S., Sneeuw, N. and Müller, J. (2014) Comparing Seven Candidate Mission Configurations for Temporal Gravity Retrieval through Full-Scale Numerical Simulation. Journal of Geodesy, 88, 31-43. http://dx.doi.org/10.1007/s00190-013-0665-9

[9] Rathke, A., et al. (2011) NG2 Team (2011) Assessment of a Next Generation Gravity Mission to Monitor the Variations of Earth’s Gravity Field. Final Report, ESTEC Contract No.: 22672/09/NL/AF.

[10] Anselmi, A., Cesare, S., Visser, P., Van Dam, T., Sneeuw, N., Gruber, T., Altes, B., Christophe, B., Cossu, F., Ditmar, P. Murboeck, M., Parisch, M., Renard, M., Reubelt, T., Sechi, G. and Texieira Da Encarnacao, J.G. (2011) NGGM Team (2011) Assessment of a Next Generation Gravity Mission to Monitor the Variations of Earth's Gravity Field. ESA Contract No. 22643/09/NL/AF, Executive Summary, Thales Alenia Space Report SD-RP-AI-0721.

[11] Reubelt, T., Sneeuw, N., Iran Pour, S., Hirth, M., Fichter, W., Müller, J., Brieden, Ph., Flechtner, F., Raimondo, J.-C., Kusche, J., Elsaka, B., Gruber, T., Pail, R., Murböck, M., Doll, B., Sand, R., Wang, X., Klein, V., Lezius, M., Danzmann, K., Heinzel, G., Sheard, B., Rasel, E., Gilowski, M., Schubert, C., Schäfer, W., Rathke, A., Dittus, H. and Pelivan, I. (2014) Future Gravity Field Satellite Missions. In: F. Flechtner, N. Sneeuw and W.-D. Schuh, Eds., Observation of the System Earth from Space-CHAMP, GRACE, GOCE and Future Missions. Geotechnologien Science Report No. 
20, Series “Advanced Technologies in Earth Sciences”, Springer, Berlin, in Press.

[12] Elsaka, B., Ilk, K.-H. and Kusche, J. (2009) Multiple Simulated Formation Flights for Future Gravity Field Recovery. Geophysical Research Abstracts, 11, 529.

[13] Massonnet, D. (1998) Roueinterferometrique. French Patent No. 339920D17306RS.

[14] Thales Alenia Space (2008) System Support to Laser Interferometry Tracking Technology Development for Gravity Field Monitoring. Summary Report, SD-RP-AI-0589.

[15] Wiese, D. (2011) Optimizing Two Pairs of GRACE-Like Satellites for Recovering Temporal Gravity Variations. PhD Thesis, Department of Aerospace Engineering Sciences, University of Colorado.

[16] Visser, P.N.A.M., Sneeuw, N., Reubelt, T., Losch, M. and van Dam, T. (2010) Space-Borne Gravimetric Satellite Constellations and Ocean Tides: Aliasing Effects. Geophysical journal International, 181, 789-805. http://dx.doi.org/10.1111/j.1365-246X.2010.04557.x

[17] Mayer-Gürr, T. (2006) Gravitationsfeldbestimmung aus der Analyse kurzer Bahnbögen am Beispiel der Satellitenmissionen CHAMP und GRACE. Ph.D. Thesis, University of Bonn, Bonn.

[18] Mayer-Gürr, T., Eicker, A., Kurtenbach, E. and Ilk, K.H. (2010) ITG-GRACE: Global Static and Temporal Gravity Field Models from GRACE Data. In: F. Flechtner, Th. Gruber, A. Gntner, M. Mandea, M. Rothacher, T. Schne and J. Wickert, Eds., System Earth via Geodetic-Geophysical Space Techniques, Springer, Berlin, 159-168. http://dx.doi.org/10.1007/978-3-642-10228-8 13 